\title{
An improved Four-Russians method and sparsified Four-Russians algorithm for RNA folding
}

\author{
Yelena Frid" and Dan Gusfield
}

\begin{abstract}
Background: The basic RNA secondary structure prediction problem or single sequence folding problem (SSF) was solved 35 years ago by a now well-known $O\left(n^{3}\right)$-time dynamic programming method. Recently three methodologies-Valiant, Four-Russians, and Sparsification-have been applied to speedup RNA secondary structure prediction. The sparsification method exploits two properties of the input: the number of subsequence $Z$ with the endpoints belonging to the optimal folding set and the maximum number base-pairs $L$. These sparsity properties satisfy $0 \leq L \leq n / 2$ and $n \leq Z \leq n^{2} / 2$, and the method reduces the algorithmic running time to $O(L Z)$. While the Four-Russians method utilizes tabling partial results.

Results: In this paper, we explore three different algorithmic speedups. We first expand the reformulate the single sequence folding Four-Russians $\Theta\left(\frac{n^{3}}{\log ^{2} n}\right)$-time algorithm, to utilize an on-demand lookup table. Second, we create a framework that combines the fastest Sparsification and new fastest on-demand Four-Russians methods. This combined method has worst-case running time of $O(\tilde{L} \tilde{Z})$, where $\frac{L}{\log n} \leq \tilde{L} \leq \min \left(L, \frac{n}{\log n}\right)$ and $\frac{Z}{\log n} \leq \tilde{Z} \leq \min \left(Z, \frac{n^{2}}{\log n}\right)$. Third we update the Four-Russians formulation to achieve an on-demand $O\left(n^{2} / \log ^{2} n\right)$-time parallel algorithm. This then leads to an asymptotic speedup of $O\left(\tilde{L} \tilde{Z}_{j}\right)$ where $\frac{Z_{j}}{\log n} \leq \tilde{Z}_{j} \leq \min \left(Z_{j}, \frac{n}{\log n}\right)$ and $Z_{j}$ the number of subsequence with the endpoint $j$ belonging to the optimal folding set.

Conclusions: The on-demand formulation not only removes all extraneous computation and allows us to incorporate more realistic scoring schemes, but leads us to take advantage of the sparsity properties. Through asymptotic analysis and empirical testing on the base-pair maximization variant and a more biologically informative scoring scheme, we show that this Sparse Four-Russians framework is able to achieve a speedup on every problem instance, that is asymptotically never worse, and empirically better than achieved by the minimum of the two methods alone.
\end{abstract}

Keywords: RNA folding, Single sequence folding, RNA secondary structure, Secondary structure prediction, FourRussians, Sparsification

\section{Background}

Non-coding RNA (ncRNA) affects many aspects of gene expression, regulation of epigenetic processes, transcription, splicing, and translation [14]. It has been observed that in eukaryotic genomes the ncRNA function is more clearly understood from the structure of the molecule, than from sequence alone. While there have been advances in methods that provide structure experimentally, the need for computational prediction has grown as the gap between sequence availability and structure has widened. In general, RNA folding is a hierarchical process in which tertiary structure folds on top of

*Correspondence: yafrid@ucdavis.edu

Department of Computer Science, UC Davis, One Shields Avenue,

Davis, CA, USA 
thermodynamically optimal ${ }^{1}$ secondary structure, secondary structure is a key component of structure prediction [14].

Efficient $O\left(n^{3}\right)$-time dynamic programming algorithms were developed more than thirty years ago to find noncrossing secondary structure of a single RNA molecule with $n$ bases [22, 23, 27, 29, 38, 39]. We call this basic folding or single sequence folding (SSF) problem. In addition, McCaskill [19] created an $O\left(n^{3}\right)$-time algorithm for the partition function for RNA secondary structure. Based on these algorithms, software has been developed and widely used [15, 16, 25, 36, 37]. Probabilistic methods, employing Stochastic context-free grammar (SFCG), were also developed to solve the basic folding problem $[7,8]$.

The accuracy of all these methods is based on the parameters given by the scoring function. Thermodynamic parameters $[17,18,28,33]$ and statistical parameters $[6,7]$, or a combination of the two $[2,13]$ are currently employed.

The Valiant [1,34], Sparsification [4, 30], and the FourRussians (FR) $[9,24]$ methods where previously applied to improve on the computation time for secondary structure prediction. For SSF, the Valiant method achieves the asymptotic time bound of $O\left(\frac{n^{3}}{2^{\operatorname{slog}(n)}}\right)$ by incorporating the current fastest $\mathrm{min} / \mathrm{max}$-plus matrix multiplication algorithm [32, 34]. The Four-Russians method was applied to single sequence [10, 24], cofolding [11] and pseudoknotted [12] folding problems. The Sparsification method, was developed to improve computation time in practice for a family of RNA folding problems, while retaining the optimal solution matrix $[4,20,21,26,30,35]$.

\section{Methods}

In this paper, we combine the Four-Russians method [24] and the Sparsification method [4]. While the former method reduces the algorithm's asymptotic running time to $\Theta\left(\frac{n^{3}}{\log ^{2} n}\right)$, the latter eliminates many redundant computations. To combine these methods, we use an ondemand tabulation (instead of a preprocessing approach which is typically applied in FR algorithms), removing any redundant computation and guaranteeing the combined method is at least as fast as each individual method, and in certain cases even faster. First, we reformulate SSF Four-Russians $\Theta\left(\frac{n^{3}}{\log ^{2} n}\right)$-time algorithm [24] to utilizes on-demand lookup table creation. Second, we combine the fastest Sparsification and Four-Russians SSF speedup methods. The Sparse Four Russians speedup presented here leads to a practical and asymptotically

${ }^{1}$ Or close to optimal. fastest combinatorial algorithm (even in the worstcase). The new algorithm has an $O(\tilde{L} \tilde{Z})$ run time where $\frac{L Z}{\log ^{2} n} \leq \tilde{L} \tilde{Z} \leq \min \left(\frac{n^{3}}{\log ^{2} n}, L Z\right)$. In practice, when accounting for every comparison operation the Sparse Four Russians outperforms both the Four-Russians and Sparsification methods. Third, we extended the FourRussian SSF algorithm to be computed in $O\left(n^{2} / \log ^{2} n\right)$ time. The simulated results for this formulation and $O(n)$ processors achieve a practice speedup on the number of comparison operations performed.

\section{Results}

\section{Problem definition and basic algorithm}

Let $s=s_{0} s_{1} \ldots s_{n-1}$ be an RNA string of length $n$ over the four-letter alphabet $\Sigma=\{A, U, C, G\}$, such that $s_{i} \in \Sigma$ for $0 \leq i<n$. Let $s_{i, j}$ denote the substring $s_{i} s_{i+1} \ldots s_{j-1}$. We note that for simplicity of exposition substring $s_{i, j}$ does not contain the nucleotide $j$. A folding (or a secondary structure) of $s$ is a set $M$ of position pairs $(k, l)$, such that: (1) $0 \leq k<l<n$; (2) and there are no two different pairs $(k, l),\left(k^{\prime}, l^{\prime}\right) \in M$ such that $k \leq k^{\prime} \leq l \leq l^{\prime}$ (i.e. each position participates in at most one pair, and the pairs are non-crossing).

Let $\beta(i, j)$ return a score associated with position pair $(i, j)$. Let $\boldsymbol{L}(s, M)$ be the score associated with a folding $M$ of RNA string $s$, and let $L(s)$ be the maximum score $\boldsymbol{L}(s, M)$ over all foldings $M$ of $s$. The RNA Folding or SSF problem is: given an RNA string $s$, compute $L(s)$, and find an optimal folding $M$ such that $L(s, M)=L(s)$. In this work, we assume the following simple scoring scheme:

$$
L(s, M)=\sum_{(i, j) \in M} \beta(i, j),
$$

where $\beta(i, j)=1$ if $\left(s_{i}, s_{j}\right) \in\{(A, U),(U, A),(C, G),(G, C)\}$, and $\beta(i, j)=0$ otherwise. Richer scoring schemes allow more biologically significant information to be captured by the algorithm. However, the algorithms for solving the problem similar recurrences and other discrete scoring schemes may be accelerated in a similar way to what we present here.

For the folding $M$ of $s_{i, j}$, an index $k \in(i, j)$ is called a split point in $M$ if for every $(x, y) \in M$, either $y<k$ or $k \leq x$. A folding $M$ is called a partitioned folding (with respect to $s_{i, j}$ ) if there exists at least one split point; otherwise $M$ is called a co-terminus folding. Let the matrix $L$ be a matrix such that $L[i, j]=L\left(s_{i, j}\right)$. In addition, let $L^{p}[i, j]$ be the maximum value of $L\left(s_{i, j}, M\right)$ taken over all partitioned foldings $M$ of $s_{i, j}$. Similarly, let $\boldsymbol{L}^{c}[\boldsymbol{i}, \boldsymbol{j}]$ be the maximum value of $L\left(s_{i, j}, M\right)$ taken over all co-terminus foldings $M$ of $s_{i, j}$. Let $L[i, i]=L[i, i+1]=0$. For all $j>i+1, L[i, j]$ can be recursively computed as follows ([23]): 


$$
\begin{aligned}
& L[i, j]=\max \left(L^{p}[i, j], L^{c}[i, j]\right), \\
& L^{p}[i, j]=\max _{k \in(i, j)}(L[i, k]+L[k, j]), \\
& L^{c}[i, j]=L[i+1, j-1]+\beta(i, j-1) .
\end{aligned}
$$

For completeness, when $j<i$, define $L[i, j]=L^{p}[i, j]=$ $L^{c}[i, j]=-\infty$.

The above recurrence may be efficiently implemented using a dynamic programming (DP) algorithm. Essentially, the DP algorithm computes and maintains values of the form $L[i, j], L^{p}[i, j]$ and $L^{c}[i, j]$ for every $0 \leq i \leq j \leq n$ in three $n+1 \times n+1$ matrices. The algorithm traverses the matrices in increasing column order index $j$ from 1 to $n$. Within each column, the cell $L[k, j]$ is computed in decreasing index order $k$ from $j-1$ to 0 . Once $L[k, j]$ is computed, $L^{p}[i, j]$ is updated for all $i<k$ such that $L^{p}[i, j]=\max \left(L^{p}[i, j], L[i, k]+L[k, j]\right)$. The solution $L(s, M)$ is stored in cell $L[0, n]$. Clearly, computing $L^{p}$ is the bottleneck of the computation, since for a given $i, j$, there may be $\Theta(n)$ split points to examine.

\section{Extending the notation and moving towards a vector by vector computation of $L$}

For a matrix $A$ and some integer intervals $I$, $J$, denote by $A[I, J]$ the sub-matrix of $A$ obtained by projecting it onto the row interval $I$ and column interval $J$. When $I=[i]$ or $J=[j]$, we simplify the notation by writing $A[i, J]$ or $A[I, j]$.

Definition 1 For a set of integers $K$, define the notation $L_{K}^{p}[i, j]$ and the max-plus operation $\otimes$ as

$L_{K}^{p}[i, j]=L[i, K] \otimes L[K, j]=\max _{k \in K}(L[i, k]+L[k, j])$.

For an interval $I=\left[i, i+1, \ldots i^{\prime}\right]$, define $L_{K}^{p}[I, j]$ to be the vector such that

$L_{K}^{p}[I, j]=L[I, K] \otimes L[K, j]=\left[L_{K}^{P}[i, j]\right.$ for all $\left.i \in I\right]$

We divide the solution matrix $L$ in two ways: $q \times q$ submatrices (Fig. 1) and size $q$ sub column vectors (the value of $q$ will be determined later). Let $\boldsymbol{K}_{\boldsymbol{g}}$ be the $g$ th interval such that $K_{g}=\{q \cdot g, q \cdot g+1, \ldots, q \cdot g+q-1\}$. We call these sets Kgroups, and use $K_{g}$ as the interval starting at index $g \cdot q$. For an index $i$, define $\boldsymbol{g}_{\boldsymbol{i}}=\left\lfloor\frac{i}{q}\right\rfloor$. It is clear that $i \in K_{g_{i}}$

Similarly, we break up the row indices into groups of size $q$, denoted by $\boldsymbol{I}_{g}$ where $I_{g}=\{k=q \cdot g, k+1$, $\ldots k+q-1\}$. (Clearly, row index set $I_{g}$ is equivalent to the Kgroup $K_{g}$. We only introduce this extra notation for simplicity of the exposition).
Given this notation $L^{P}[i, j]$ can be rewritten as maximization $L_{K_{g}}^{p}[i, j]$ values for all $K_{g}$ index Kgroups between $i$ and $j$. However, in some cases, the indices $\left\{i+1, \ldots q \cdot g_{i+1}-1\right\}$ do not form a full Kgroup $K_{g_{i}}$. Similarly indices $\left\{q g_{j}, q g_{j}+1, \ldots j-1\right\}$ do not form a full Kgroup $K_{g_{j}}$. Therefore, $L^{P}[i, j]$ can be computed by maximizing the full and non full Kgroups $K_{g}$. In Eq. 4 and the following sections we do not explicitly differentiate between full and non full groups.

$$
L^{p}[i, j]=\max _{g_{i} \leq g \leq g_{j}} L_{K_{g}}^{p}[i, j]
$$

We extend the notation further, to compute the matrix $L^{p}$ not cell by cell but instead by vectors of size $q$ corresponding to the $I_{g^{\prime}}$ row sets, as follows.

$$
L^{p}\left[I_{g^{\prime}}, j\right]=\max _{g^{\prime} \leq g^{\prime} \leq g_{j}} L_{K_{g}}^{p}\left[I_{g^{\prime}}, j\right] .
$$

The DP algorithm can be updated to incorporate the extended notation. Within each column, compute the matrices in vectors of size $q$. Once $L\left[K_{g}, j\right]$ is computed it is used in computation of $L_{K_{g}}^{p}\left[I_{g^{\prime}}, j\right]$ for $g^{\prime}<g$. When computing $L_{K_{g^{\prime}}}^{p}\left[I_{g^{\prime}}, j\right]$ we follow Eq. $1-3$ to complete the computation of cells $L\left[I_{g^{\prime}}, j\right]$.

\section{Sparsification of the SSF algorithm}

The Sparsification method achieves a speedup by reducing the number of split points examined during the computation of $L^{P}[i, j]$. As Fig. 2 shows the focus of Sparsified Four Russians algorithm will narrow down only on those submatrices whose split points are step-oct for a particular $i, j[4,30]$.

\section{OCT and STEP sub-instances of sequence s}

Sub-instance $s_{i, j}$ is optimally co-terminus (OCT) if every optimal folding of $s_{i, j}$ is co-terminus. We introduce the extra notation below

if $L[i, j]=L^{c}[i, j]>L^{p}[i, j]$ then we say $L[i, j]$ is OCT.

Sub-instance $s_{i, j}$ is STEP, if $L[i, j]>L[i+1, j]$ where $L[i, j]=L\left(s_{i, j}\right)$ and $L[i+1, j]=L\left(s_{i+1, j}\right)$. For ease of exposition we also say $L[i, j]$ is $S T E P$ when $s_{i, j}$ is $S T E P$. A $S T E P$ sub-instance $s_{i, j}$ implies that nucleotide $i$ is paired in every optimal folding of $s_{i, j}$.

Fact 1 For every sub-instance $s_{i, j}$ with $j>i$ there is an optimal split point $k \in(i, j)$ such that either $k=i+1$ or $L[i, k]$ is $S T E P$ and $L[k, j]$ is $O C T$ [4].

Notation: For the index set $K=\left\{k, k+1, \ldots k^{\prime}\right\}$ and column $j$, let $K^{o c t_{j}}$ be the set of indices such that $K^{\text {oct }}{ }_{j} \subset K$ and $\forall_{k \in K^{o c t} t_{j}} L[k, j]$ is OCT. Given the row interval $I=\left\{i, i+1, \ldots i^{\prime}\right\}$, let $I^{s t e p_{k}}$ be the set of rows such that $I^{\text {step }}$ $_{k} \subset I$, and for all $i \in I^{\text {step }}{ }_{k} L[i, k]$ is STEP. 


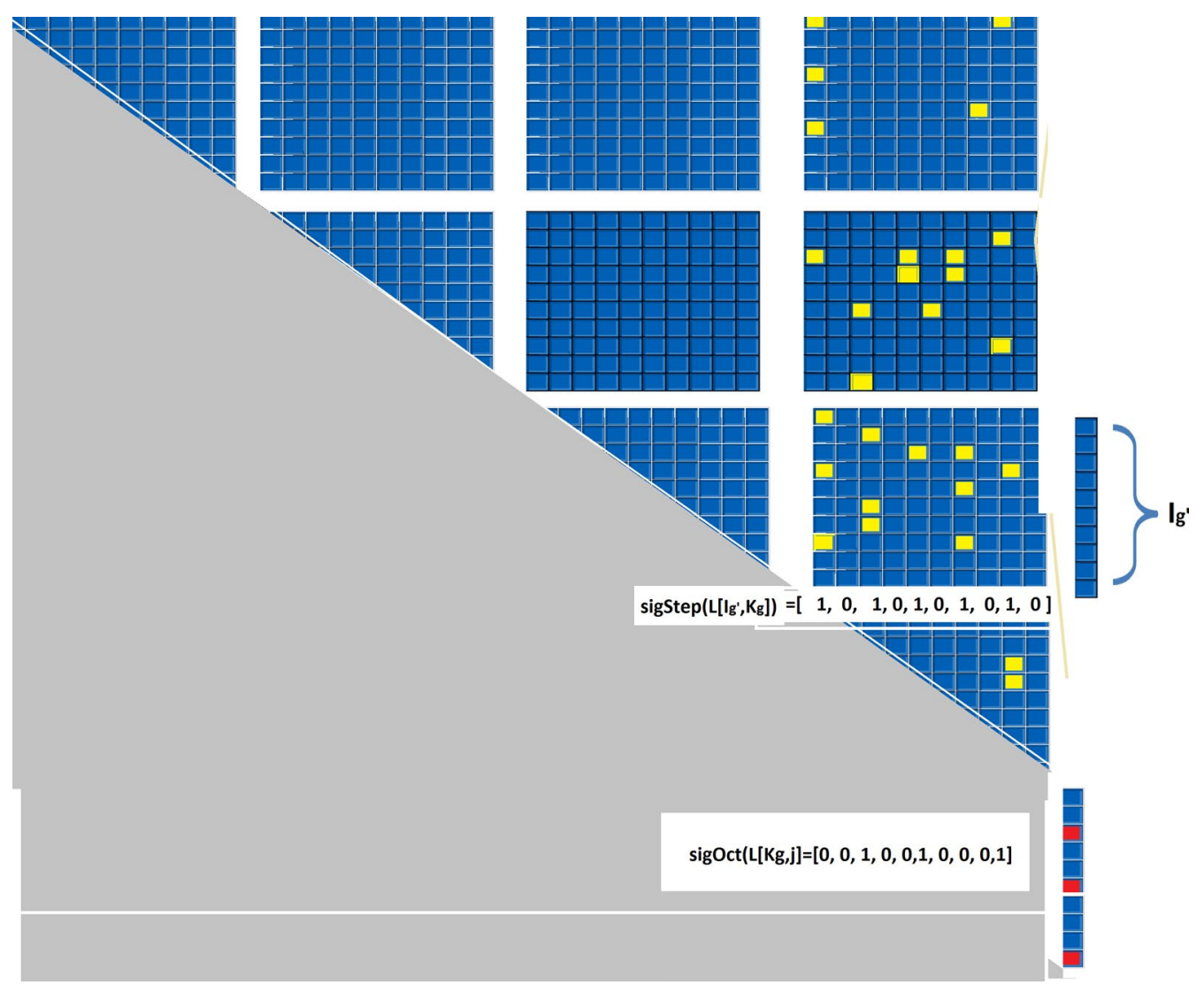

Fig. 1 An example of how a solution matrix $L$ is broken down into submatrices. Using the extended vector notation we can say that cell $L[i, j]$ belongs to the vector $L\left[K_{g_{i}}, j\right]$ as well as submatrix $L\left[g_{g_{i}}, K_{g_{j}}\right]$. We partition the solution matrix $L$ into $O\left(n^{2} / q\right)$ vectors of size $O(q)$ and $O\left(n^{2} / q^{2}\right)$ submatrices, of size $O\left(q^{2}\right)$

We further define operation $\otimes_{\text {step-oct }}$ such that given $I=\left\{i, i+1, \ldots, i^{\prime}\right\}$ and $K=\left\{k, k+1, \ldots, k^{\prime}\right\}$, $L[I, K] \otimes_{\text {step-oct }} L[K, j] \quad$ results in $A\left[\begin{array}{ll}I, & j\end{array}\right]$ where $\forall_{i \in\left(I^{\text {step }} \mathrm{F}_{k} \mathrm{Itep}_{k+1} \cup \ldots I^{\text {step }} k^{\prime}\right)} A[i, j]$ is computed by the following procedure:

$$
\begin{aligned}
& \text { for } k \in K^{\text {oct }_{j}} \text { do } \\
& \forall_{i \in I^{s t e p_{k}}} \quad A[i, j]=\max (L[i, k]+L[k, j], A[i, j])
\end{aligned}
$$

Using the operation $\otimes_{\text {step-oct }}$ and based on Fact 1 . We reduce the time to compute $L^{p}\left[I_{g^{\prime}}, j\right]$ by considering a split-point $k$ only if $k=i+1$ or $L[i, k]$ is STEP and $L[k, j]$ is OCT for $i \in I_{g^{\prime}}$ and $k \in(i, j)$.

$L^{p}\left[I_{g^{\prime}}, j\right]=\max _{g^{\prime} \leq g \leq g_{j}} L_{K_{g}}^{p}\left[I_{g^{\prime}}, j\right]=\max _{g^{\prime} \leq g \leq g_{j}} L\left[I_{g^{\prime}}, K_{g}\right] \otimes_{\text {step-oct }} L\left[K_{g}, j\right]$

Note Eq. 6 does not explicitly show that for $L_{K_{g^{\prime}}}^{P}\left[I_{g^{\prime}}, j\right]$ the split-point $i+1$ must be examined for every $i \in I_{g^{\prime}}$.

Asymptotic time bound of sparsified SSF When computing matrix $L^{p}[i, j]$ we examine value $L[i, k]$ only if $L[k, j]$ is $O C T$. Let $Z$, be the total number of sub-instances in $s$ or cells in matrix $L$ that are $O C T$. Given that $L[k, j]$ is $O C T, L^{p}[i, j]$ must examine the split point $k$, for all $i \in\{0,1, \ldots k\}$ such that $L[i, k]$ is STEP. Let $\boldsymbol{L}$ be the total number of STEP sub-instances in column $k$. More precisely $L=\left|\{0,1, \ldots k\}^{\text {step }_{k}}\right|$ (Creating the list of splitpoints that correspond to STEP incidence requires no additional computation time [4]). The total time to compute SSF when examining only STEP, OCT combinations (Sparsification method), is $O(L Z)$. As shown in Backofen et al. [4] $Z$ is bounded by $Z \leq n^{2}$ and $L$ is bounded by $L \leq \frac{n}{2}$. The overall asymptotic time bound of the Sparsification method is $O(L Z)$ remains $O\left(n^{3}\right)$.

\section{On-demand Four Russians speedup}

Presented here is an on-demand version of the $\Omega\left(\log ^{2} n\right)$ -time Four-Russians algorithm implied by Pinhas et al. [24].

Observation 1 The scores stored in $L[k, j]$ and $L[k+1, j]$ differ by the effect of adding only one more nucleotide (i.e., $\left.s_{k}\right)$. Therefore, $L[k, j]-L[k+1, j]$ belongs to a finite set of 


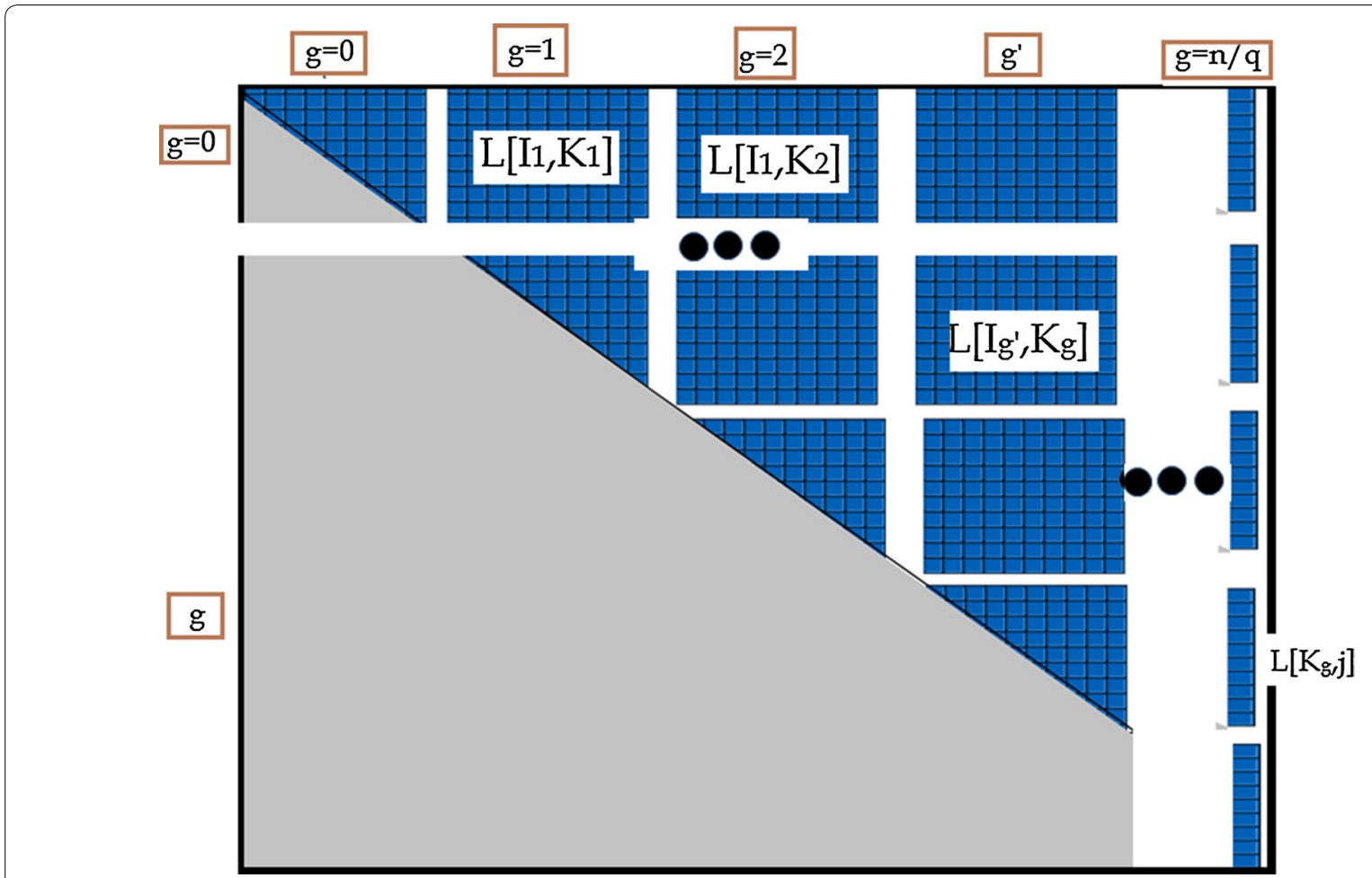

Fig. 2 An sample examination to determine wether a submatrix and vectors are STEP-OCT. The yellow cells indicate STEP instances. The red cells indicate OCT instances. The $L\left[I_{g^{\prime}}, K_{g}\right] \otimes L\left[K_{g}, j\right]$ operation is only performed on submatrices with sigSTEP $\odot$ sigOCT $>0$

differences $\mathbb{D}$, where $\mathbb{D}$ is the set of scores created as the result of the scoring scheme $\beta$. The cardinality of the set of differences, $D=|\mathbb{D}|$, is $O(1)$ when $\beta$ is discrete. For the simple $\beta$ scoring function ( +1 for every permitted pair, and 0 otherwise), the set $\mathbb{D}$ is equal to $\{0,1\}$ and therefore $|\mathbb{D}|=2[23]$.

Let $\vec{x}=\left[x_{0}, x_{1}, \ldots, x_{q-1}\right]$ be an integer vector of length $q$. We say that $\vec{x}$ is $D$-discrete if $\forall_{l \in(0, q)}\left|x_{l-1}-x_{l}\right| \in \mathbb{D}$. We define the $\Delta$-encoding of 2-discrete vector $\vec{x}$ to be a pair of integers $\left(x_{0}, \Delta_{x}\right)$ such that $x_{0}$ is the first element in $\vec{x}$ and $\Delta_{x}$ is the integer representation of the binary vector $\left[x_{0}-x_{1}, x_{1}-x_{2}, \ldots, x_{q-2}-x_{q-1}\right]$. Note that $0 \leq \Delta_{x}<2^{q-1}$. For simplicity, we will interchangeably use $\vec{x}$ to imply either $\left(x_{0}, \Delta_{x}\right)$ or $\left[x_{0}, x_{1}, \ldots, x_{q-1}\right]$. Clearly, $\Delta$-encoding takes $O(q)$ time to compute.

$\Delta$-encoding vector operations:

- Let $\left(x_{0}, \Delta_{\vec{x}}\right)+c=\left(x_{0}+c, \Delta_{\vec{x}}\right)$ be equivalent to $\vec{x}+c=\left[x_{0}+c, x_{1}+c, \ldots, x_{q-1}+c\right]$.

- Let $B \otimes\left(x_{0}, \Delta_{x}\right)$ be equivalent to $B \otimes \vec{x}$.

- Let $\max \left(\left(x_{0}, \Delta_{x}\right),\left(y_{0}, \Delta_{y}\right)\right)$ be equivalent to $\max (\vec{x}, \vec{y})$.

\section{MUL lookup table}

Based on Observation 1, any column vector in matrix $L$ is 2-discrete. Given vector $L\left[K_{g}, j\right]$ and its $\Delta$-encoding $\left(x_{0}=L[g q, j], \quad \Delta_{x}=\Delta_{L\left[K_{g}, j\right]}\right), \quad$ it is clear that $\Delta_{x} \in\left[0,2^{q}-1\right]$.

Fact $22 L\left[I_{g^{\prime}}, K_{g}\right] \otimes L\left[K_{g}, j\right]$ is equivalent to $L\left[I_{g^{\prime}}, K_{g}\right] \otimes\left(0, \Delta_{L\left[K_{g}, j\right]}\right)$ $+L[g q, j][24]$.

Let $M U L_{B}[i]$ be a lookup table, where given a $q \times q \quad$ submatrix $B=L\left[I_{g^{\prime}}, K_{g}\right]$ and $i=\Delta_{L\left[K_{g}, j\right]}$, the entry $M U L_{L\left[I_{g^{\prime}}, K_{g}\right]}\left[\Delta_{L\left[K_{g}, j\right]}\right]=\left(y_{0}, \Delta_{y}\right) \quad$ where $\vec{y}=L\left[I_{g^{\prime}}, K_{g}\right] \otimes\left(0, \Delta_{L\left[K_{g}, j\right]}\right)$. We could reformulate the computation of $L_{K_{g}}^{p}\left[I_{g^{\prime}}, j\right]$ to utilize the MUL lookup table.

$$
\begin{aligned}
L_{K_{g}}^{p}\left[I_{g^{\prime}}, j\right] & =L\left[I_{g^{\prime}}, K_{g}\right] \otimes L\left[K_{g}, j\right] \\
& =M U L_{L\left[I_{g^{\prime}}, K_{g}\right]}\left[\Delta_{L\left[K_{g}, j\right]}\right]+L[g q, j] .
\end{aligned}
$$

Equation 7, abstracts the detail that we still have to compute each referenced entry in the MUL lookup table. Each entry in the MUL lookup table is computed on-demand i.e. only when it corresponds to a required calculation. (This removes any extraneous calculation incurred when 
preprocessing all possible entries as in the typical FourRussians implementation.) If entry $M U L_{L\left[I_{g^{\prime}}, K_{g}\right]}\left[\Delta_{L\left[K_{g}, j\right]}\right]$ does not exist we compute $L\left[I_{g^{\prime}}, K_{g}\right] \otimes\left(0, \Delta_{L\left[K_{g}, j\right]}\right)$ directly in $O\left(q^{2}\right)$ time. If entry $M U L_{L\left[I_{g^{\prime}}, K_{g}\right]}\left[\Delta_{L\left[K_{g}, j\right]}\right]$ exists then the operation is $O(1)$-time lookup.

There are $O\left(\frac{n^{2}}{q^{2}}\right)$ submatrices within $L$. For each submatrix the maximum number of entries we compute for lookup table $M U L$ is $2^{q-1}$. In total, the asymptotic time bound to populate lookup table $M U L$ is $O\left(\frac{n^{2}}{q^{2}} \cdot 2^{q-1} \cdot q^{2}\right)=O\left(n^{2} \cdot 2^{q}\right)$

\section{MAX lookup table}

Let the $\max$ of two 2-discrete $q$-size vectors $\vec{v}$ and $\vec{w}$, denoted $\max (\vec{v}, \vec{w})$, result in a $q$-size vector $\vec{z}$, where $\forall_{0<k<q} z_{k}=\max \left(v_{k}, w_{k}\right)$. Without loss of generality, let $w_{0} \geq v_{0}$. Comparing the first element in each vector there are two possibilities either (1) $w_{0}-v_{0}>q-1$ or (2) $w_{0}-v_{0} \leq q-1$. In the first case, $\left(w_{0}-v_{0}>q-1\right)$, it is clear that $\max (\vec{v}, \vec{w})$ is equal to $\vec{w}$. In the second case, we make use of the following fact [24].

Fact 3 Given two vectors $\left(w_{0}, \Delta_{w}\right)$ and $\left(v_{0}, \Delta_{v}\right)$, if $w_{0}-v_{0} \leq q-1$ then $\max (\vec{v}, \vec{w})=\max \left(\left(0, \Delta_{v}\right),\left(w_{0}-v_{0}\right.\right.$, $\left.\left.\Delta_{w}\right)\right)+v_{0}$.

Lets define lookup table $M A X$ such that entry $M A X\left[i, i^{\prime}, h\right]=\max \left((0, i),\left(h, i^{\prime}\right)\right)$. Hence, we reformulate Fact 3. to incorporate the $M A X$ lookup table:

$$
\max (\vec{v}, \vec{w})=\operatorname{MAX}\left[\Delta v_{0}, \Delta w_{0},\left(w_{0}-v_{0}\right)\right]+v_{0}
$$

We summarize these results in the function $\Delta$ max:

\section{Function $\Delta$ max : :}

input: $\vec{v}, \vec{w}$ such that $w_{0} \geq v_{0}$ and $\vec{v}=\left(v_{0}, \Delta_{v}\right)$ and $\vec{w}=\left(w_{0}, \Delta_{w}\right)$ output: $\vec{z}=\left(z_{0}, \Delta_{z}\right)$ where $\forall_{i \in[0, q)} z_{i}=\max \left(v_{i}, w_{i}\right)$

$\operatorname{if}\left(w_{0}-v_{0} \geq q-1\right): \quad \vec{z}=\vec{w}$

else : $\quad \vec{z}=\operatorname{MAX}\left[\Delta v_{0}, \Delta w_{0},\left(w_{0}-v_{0}\right)\right]+v_{0}$

In Eq. 8, below, we integrate the vector comparison function $\Delta$ max. Each vector $L^{p}\left[I_{g^{\prime}}, j\right]$ is computed by maximizing over $O(n / q)$ vectors. We will compute the lookup table $M A X$ on-demand for every entry that does not exist an $O(q)$. Clearly the lookup table $M A X$ will contain at most $2^{(q-1)} \cdot 2^{(q-1)} \cdot q$ for all entries. In worst case, the lookup table $M A X$ computes in $O\left(2^{q^{2}} q\right)$ time.

$L^{p}\left[I_{g^{\prime}}, j\right]=\underset{g^{\prime} \leq g \leq g_{j}}{\Delta \max }\left(M U L_{L\left[I_{g^{\prime}}, K_{g}\right]}\left[\Delta_{L\left[K_{g}, j\right]}\right]+L[g q, j]\right)$

The matrix $L^{p}$ and hence $L$ is solved by a total of $O\left(\frac{n^{2}}{q}\right)$ computations of Eq. 8. In total, given lookup table $M U L$ and $M A X$, the time to compute the Four-Russians SSF is

$$
O(\underbrace{\frac{n^{3}}{q^{2}}}_{\text {computation }}+\underbrace{2^{2 q} q+n^{2} 2^{q}}_{\text {on-demand lookup table }}) \text {. }
$$

Setting $q=\epsilon \log n$, where $\epsilon \in(0, .5)$ [31], the total computation time is equal to $\Theta\left(\frac{n^{3}}{\log ^{2} n}\right)$, which achieves a speedup by a factor of $\Omega\left(\log ^{2} n\right)$, compared to the original $O\left(n^{3}\right)$-time solution method.

\section{Extending to $D$-discrete vectors}

We define the $\Delta$-encoding of $D$-discrete vector $\vec{x}$ to be a pair of integers $\left(x_{0}, \Delta_{x}\right)$ such that $x_{0}$ is the first element in $\vec{x}$ and $\Delta_{x}$ is the integer representation in base 10 of the vector $\left[x_{0}-x_{1}, x_{1}-x_{2}, \ldots, x_{q-2}-x_{q-1}\right]$ in base $\mathrm{D}$ where $x_{0}$ is the most significant integer. Note that $0 \leq \Delta_{x}<D^{q-1}$. As a result for a more complicated scoring scheme $B$ we could apply the Four-Russians speedup by augmenting the encode, and decode functions as well as the $\Delta$ max algorithm.

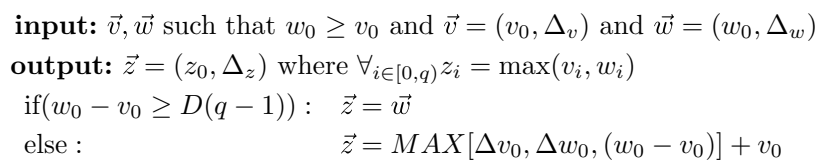

This would result in a total asymptotic time for FourRussians SSF where $|D|>2$ of

$$
O(\underbrace{\frac{n^{3}}{q^{2}}}_{\text {computation }}+\underbrace{D^{2 q} q+n^{2} D^{q}}_{\text {on-demand lookup table }}) .
$$

Setting $q=\epsilon \log _{D} n$, where $\epsilon \in(0, .5) \quad$ [31], the total computation time is equal to $\Theta\left(\frac{n^{3}}{\log ^{2} n}\right)$, which achieves a speedup by a factor of $\Omega\left(\log ^{2} n\right)$, compared to the original $O\left(n^{3}\right)$-time solution method.

\section{Sparse Four-Russian method}

With the Four-Russians method, a speedup is gained by reducing $q$ split point index comparisons for $q$ subsequences to a single $O(1)$ time lookup. The Sparsification method reduces the comparison to only those indices which correspond to $S T E P-O C T$ folds.

\section{STEP-OCT condition for sets of split points}

In this section, we achieve a Sparsified Four-Russian speedup for the computation of the $L^{p}$ matrix. As in the Four Russians method, we will conceptually break up the 
solution matrix $L$ in two ways: in $q \times q$ size submatrices, and $q$ size subcolumn vectors. The submatrices are indexed by $g^{\prime}$ and $g$ such that the corresponding submatrix is $L\left[I_{g^{\prime}}, K_{g}\right]$. The subcolumn vectors are indexed by $g$ and $j$, such that the corresponding subcolumn vector is $L\left[K_{g}, j\right]$.

We augment the Four-Russians SSF to reduce the number of entries, and lookups into the $M U L$ table. If and only if, the matrix $L\left[I_{g^{\prime}}, K_{g}\right]$ contains at least one cell $L[i, k]$ that is STEP and within vector $L\left[K_{g}, j\right]$ the cell $L[k, j]$ is OCT we will lookup $M U L_{L\left[I_{g^{\prime}}, K_{g}\right]}\left[\Delta_{L\left[K_{g}, j\right]}\right]$. If such an entry does not exist we will compute $L\left[I_{g^{\prime}}, K_{g}\right] \otimes\left(0, \Delta_{L\left[K_{g}, j\right]}\right)$ and store the result into lookup table $M U L$.

The following notation will be used to help determine if a split point Kgroup should be examined in the computation.

OCT subcolumn vector Given the vector $L\left[K_{g}, j\right]$ let $\vec{m}$ be a $q$ size binary vector such that $\forall_{0 \leq x \leq q-1} m[x]=1$ if $L[g q+x, j]$ is $O C T$. Let the sigOct of the vector $L\left[K_{g}, j\right]$, written $\operatorname{sigOct}\left(L\left[K_{g}, j\right]\right)$, be equal to $m$ the integer representation of the binary vector $\vec{m}$. Clearly $0 \leq m<2^{q}$, and if $m>0$ then $L\left[K_{g}, j\right]$ contains at least one $O C T$ instance. Let $O(\tilde{Z})$ be the total number of subcolumn vectors which contain an instance that is $O C T$. Clearly, $\frac{Z}{q} \leq \tilde{Z} \leq \min \left(\frac{n^{2}}{q}, Z\right)$.

STEP submatrix Given the submatrix $L\left[I_{g^{\prime}}, K_{g}\right]$, let $\vec{m}^{\prime}$ be a $q$ size binary vector such that $\forall_{x \in[0, q)} m^{\prime}[x]=1$ if $\exists_{0 \leq i \leq q-1} L\left[q g^{\prime}+i, q g+x\right]$ is STEP. Let sigStep of a submatrix, written $\operatorname{sigStep}\left(L\left[I_{g^{\prime}}, K_{g}\right]\right)$, be equal to $m^{\prime}$ the integer representation of the binary vector $\vec{m}^{\prime}$. Clearly $0 \leq m^{\prime}<2^{q}$. Let $\tilde{L}$ be the total number of submatrices which contain an instance that is STEP within $L\left[[0, n], K_{g}\right]$. Clearly, $\frac{L}{q} \leq \tilde{L} \leq \min \left(\frac{n}{q}, L\right)$.

Observation 2 Suppose that, $s_{i, k}$ is STEP, and integer $m^{\prime}=\operatorname{sigStep}\left(L\left[I_{g^{\prime}}, K_{g}\right]\right)$ such that $i \in I_{g^{\prime}}\left(\right.$ or $\left.I_{g^{\prime}}=I_{g_{i}}\right)$ and $k \in K_{g}$ (or $K_{g}=K_{g_{k}}$ ). Then, the corresponding binary vector $\vec{m}^{\prime}$ must be set to 1 in position $x$ where $x$ is an index such that $k=q g+x$. More precisely, if $L[i, k]$ is STEP then $m^{\prime}[x]=1$ by the definition of sigStep.

Observation 3 Suppose $s_{k, j}$ is OCT, and suppose integer $m=\operatorname{sigOct}\left(L\left[K_{g}, j\right]\right)$ such that $k \in K_{g}$. Then, the corresponding binary vector $\vec{m}$ must be set to 1 in position $x$, where $x$ is an index such that $k=q g+x$. More precisely, if $s_{k, j}$ is OCT then $m[x]=1$ by the definition of sigOct.

Given two binary vectors $v$ and $w$ the dot product of their integer representation is equal to a binary number $x$ such that $x=v \odot w=v_{0} \wedge w_{0} \vee v_{1} \wedge w_{1} \vee \ldots$ $\vee v_{q-1} \wedge w_{q}$ where $|v|=|w|=q-1$
Theorem 1 For any subinstance $s_{i, j}$ either $i+1$ is the optimal split point, or there is an optimal split point $k \in(i, j)$, such that $\operatorname{sigStep}\left(L\left[I_{g_{i}}, K_{g_{k}}\right]\right) \odot \operatorname{sigOct}\left(L\left[K_{g_{k}}, j\right]\right)$ equals 1 .

Proof Based on Fact 1 for any sub-instance $s_{i, j}$ there is an optimal split point $k$ such that either $k=i+1$ or $s_{i, k}$ is STEP and $s_{k, j}$ is OCT. If $s_{i, k}$ is STEP and $s_{k, j}$ is $O C T$ then $L[i, k]$ is STEP and $L[k, j]$ is OCT. The cell $L[i, k]$ belongs to submatrix $L\left[I_{g_{i}}, K_{g_{k}}\right]$ and the cell $L[k, j]$ belongs to the vector $L\left[K_{g_{k}}, j\right]$. Let $x$ be an index such that $k=q g_{k}+x$. Let $\vec{m}^{\prime}$ be a binary vector that corresponds to $\operatorname{sigStep}\left(L\left[I_{g_{i}}, K_{g_{k}}\right]\right)$. Based on Observation $2, m^{\prime}[x]$ must equal 1 . Let $\vec{m}$ be the binary vector that corresponds to $\operatorname{sigOct}\left(L\left[K_{g_{k}}, j\right]\right)$. Based on Observation $3, m[x]$ equals 1 . Therefore, $m[x] \wedge m^{\prime}[x]=1$ and $\operatorname{sigStep}\left(L\left[I_{g_{i}}, K_{g}\right]\right) \odot \operatorname{sigOct}\left(L\left[K_{g}, j\right]\right)=1$.

Notation: The index $g$ is STEP-OCT if given the set of rows $I_{g^{\prime}}$ and the column $j$ if $\operatorname{sigStep}\left(L\left[I_{g^{\prime}}, K_{g}\right]\right) \odot$ $\operatorname{sigOct}\left(L\left[K_{g}, j\right]\right)=1$.

We can reformulate the computation of $L^{p}\left[I_{g^{\prime}}, j\right]$ by referencing the lookup table $M U L$ only if $g$ is STEP-OCT. This reduces the number of operations used in computing the bottleneck $L^{P}$ matrix.

$$
\begin{aligned}
& L^{p}\left[I_{g^{\prime}}, j\right]=\underset{g}{\Delta} \max _{\operatorname{sis} S T E P-O C T}\left(M U L_{L\left[I_{g^{\prime}}, K_{g}\right]}\left[\Delta_{L\left[K_{g}, j\right]}\right]+L[g q, j]\right) \\
& \text { where } g \in\left[g^{\prime}, g_{j}\right]
\end{aligned}
$$

We update the DP algorithm to only access the $M U L$ lookup table for matrix and vector combinations that satisfy the property

$$
\operatorname{sigStep}\left(L\left[I_{g^{\prime}}, K_{g}\right]\right) \odot \operatorname{sigOct}\left(L\left[K_{g}, j\right]\right)=1 \text {. }
$$

Let $G$ be a lookup table, where give an index $g \in[0, n / q]$ and integer $m \in\left[0,2^{q}\right]$ the $G[g][m] \subset\left\{I_{0}, I_{1}, \ldots, I_{g}\right\}$ is a set of row index intervals. Each index $I_{g^{\prime}}$ within G[g][m] satisfies the following condition:

$$
\text { if } I_{g^{\prime}} \in G[g][m] \text { then } \operatorname{sigStep}\left(L\left[I_{g^{\prime}}, K_{g}\right]\right) \odot m=1 .
$$

Lookup table $G$ (updated on-demand) allows us to implement Eq. 9. As $L\left[K_{g}, j\right]$ is computed, the corresponding SigOct is also computed. Let $m=\operatorname{sigOct}\left(L\left[K_{g}, j\right]\right)$. By iterating through $I_{g^{\prime}} \in G[g][m]$ set of row indices we access table $M U L$ only when both of the following conditions hold at the same time: the submatrix $L\left[I_{g^{\prime}}, K_{g}\right]$ contains at least one cell $L[i, k]$ where $s_{i, k}$ is $S T E P$ and within vector $L\left[K_{g}, j\right]$ the cell $L[k, j]$ contains $s_{k, j}$ that is OCT (where $i \in I_{g^{\prime}}$ and $k \in K_{g}$ ).

The Sparsified Four-Russian algorithm implements Eq. 9. The complete function will tabulate $S T E P$, and $O C T$ instances as well as sigStep and sigOct values. The G, MUL and $M A X$ lookup tables will be computed on-demand. 
Table 1 Number of all comparisons computed

\begin{tabular}{lllll}
\hline Size & $\boldsymbol{O}\left(\boldsymbol{n}^{\mathbf{3}}\right)$ & FR & SP & SFR \\
\hline 64 & 43,680 & 12,014 & 2733 & 1837 \\
128 & 349,504 & 49,456 & 13,196 & 9982 \\
256 & $2,796,160$ & 346,692 & 79,544 & 41,393 \\
512 & $22,500,863$ & $5,746,853$ & 650,691 & 503,425 \\
\hline
\end{tabular}

Four-Russians (FR) algorithm. In all test cases, the Sparse Four-Russians performed better than the minimum of either method alone.

\section{An $O\left(n^{2} / \log ^{2}(n)\right)$ simple parallel Four-Russians RNA folding algorithm}

Lets solve the recurrence relation (Eq. 1-3) in increasing index $j$ order and then move up the column $j$ computing one cell at a time in decreasing $i$ order. Each cell $L[i, j]$ is solved by calculating Eq. $1-3$ for all $i<k \leq j$.

Given this $j, i, k$ order, let us reformulate the computation by moving up each column in $O(n / q) q$-size subcolumn vectors instead of $n$ cells.

\section{Utilizing $n$ processors}

Lets create a new process for each column $j$, creating $n$ process in total. We can synchronously move up the matrix computing each column subvector such that on iteration $d$ we compute $L\left[I_{g_{j}-d}, j\right]$ for all $j \in(0, n)$.

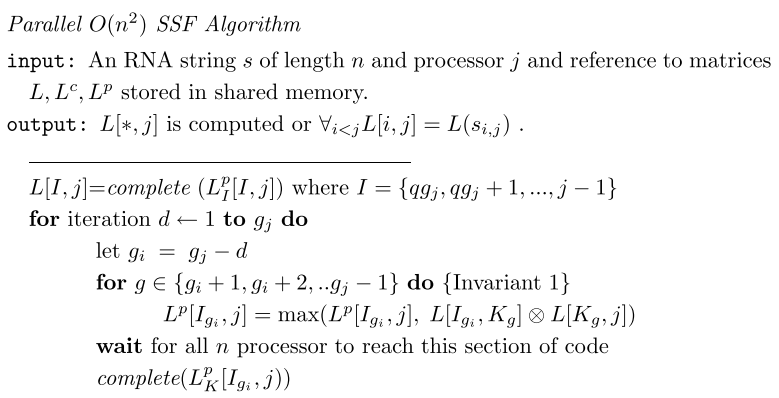

Invariant 1 Given $g_{i}$ and $g_{j} \forall_{i \in I_{g_{j}}} \forall_{k \in K_{g}} L[i, k]=L\left(s_{i, k}\right)$. In other words, submatrix $L\left[I_{g_{i}}, K_{g}\right]$ is computed. Similarly $L\left[K_{g}, j\right]$ is computed or $\forall_{k \in K_{g}} L[k, j]=L\left(s_{k, j}\right)$.

Please note that the function complete assumes that $L_{\bar{K}}^{p}[I, j]$ is computed, where $\bar{K}=\{i, i+1, \ldots j-2, j-1\}-K$.

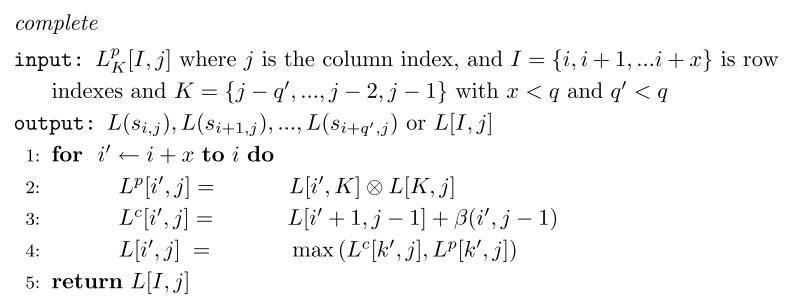

Replacing the $\left.\max \left(L^{p}\left[I_{g_{i}}, j\right], L\left[I_{g_{i}}, K_{g}\right]\right) \otimes L\left[K_{g}, j\right]\right)$ computation with lookups into $M U L$ and $M A X$ tables would reduces the run-time for finding the solution matrix $L$ to $O\left(n^{2} / \log ^{2} n\right)$. As stated in "Extending to D-discrete vectors" section it is possible to create lookup tables on-demand and achieve a reduction in computation time of $\Omega\left(\log ^{2} n\right)$ factor.

The preprocessing can also be achieve in parallel reducing the asymptotic cost form $O\left(n^{3} / \log ^{2} n\right)$ to $O\left(n^{2} / \log ^{2} n\right)$. If entry $M U L_{L\left[I_{g_{i}}, K_{g}\right]}\left[\Delta_{L\left[K_{g}, j\right]}\right]$ does not exist we compute $L\left[I_{g_{i}}, K_{g}\right] \otimes\left(0, \Delta_{L\left[K_{g}, j\right]}\right)$ directly in $O\left(q^{2}\right)$.

There are $O\left(\frac{n^{2}}{q^{2}}\right)$ submatrices within $L$. For each submatrix the maximum number of entries we compute for lookup table $M U L$ is $D^{q-1}$. However, in each iteration at worse $O(n)$ of the entries are computed simultaneously. In total, the asymptotic time bound to populate lookup table $M U L$ is $O\left(\frac{\frac{n^{2}}{q^{2}} \cdot D^{q-1} \cdot q^{2}}{n}\right)=O\left(\frac{n^{2} \cdot D^{q}}{n}\right)=O\left(n \cdot D^{q}\right)$.

Based on Williams [31] $O\left(D^{q}\right)$ is bound by $O\left(n / \log ^{2} n\right)$ when setting $q=\epsilon \log n$. Hence, for the MUL lookup table the total asymptotic computation time is $O\left(n \cdot D^{q}\right)=O\left(n^{2} / \log ^{2} n\right)$, For the MAX table similarly the serial computation of $O\left(D^{2 q} q\right)$ total time is reduced by a factor of $n$ in the parallel case. The total computation time for the $M A X$ table is therefore $O\left(n / \log ^{3} n\right)$.

Parallel Four-Russian Folding s

input: An RNA string $s$ of length $n$ and processor $j$ and reference to matrices $L, L^{c}, L^{p}$ stored in shared memory.

output: $L[*, j]$.

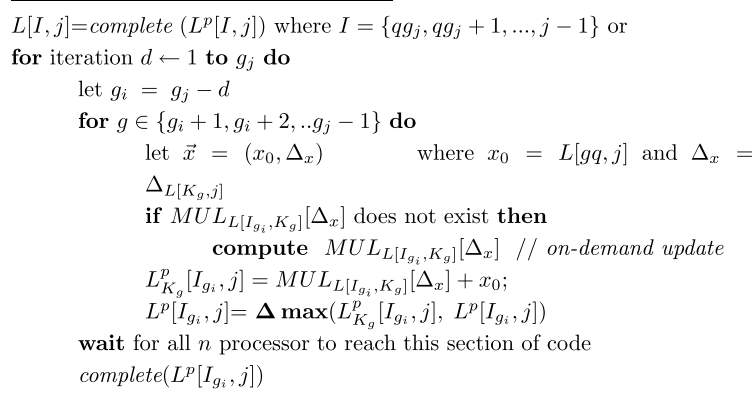




\section{Parallel sparisified Four-Russians single sequence folding algorithm}

Let $Z_{x}$ be the number of OCT cells in column $x$. Let $\forall_{x \in[0, n]} Z_{j} \geq Z_{x}$.

The parallel algorithm would take as long as would take as a it takes for the last processor to complete.

In order to extend the parallel Four-Russians single sequence folding algorithm to utilize the Sparsification speedup we will limit the call to $M U L$ table only if $\operatorname{sigSTEP}\left(L\left[I_{g_{i}}, K_{g}\right]\right) \odot \operatorname{sigOCT}\left(L\left[K_{g}, j\right]\right)>0$. As result given $Z_{j}$ the total time to compute for processor $j$ is $O\left(\tilde{L} \tilde{Z}_{j}\right)$ where $\frac{Z_{j}}{\log n} \leq \tilde{Z}_{j} \leq \min \left(Z_{j}, \frac{n}{\log n}\right)$.

\section{Conclusion}

This work combines the asymptotic speedup of FourRussians with the very practical speedup of Sparsification. The on-demand formulation of the Four-Russians not only removes all extraneous computation. This approach allows the Four-Russians SSF to achieve a speedup in practice for realistic scoring schemes. This also leads us to take advantage of the sparsity properties. Through asymptotic analysis and empirical testing on the base-pair maximization variant and a more biologically informative scoring scheme, we show that the Sparse Four-Russians framework is able to achieve a speedup on every problem instance, that is asymptotically never worse, and empirically better than achieved by the minimum of the two methods alone. We also showed that through some re-organization we could apply the Four-Russians speedup to parallel algorithm and achieve and asymptotic time of $O\left(n^{2} / \log ^{2} n\right)$. The algorithm created here can be implemented in CUDA to compute on multiprocessor GPUs. Because the algorithm allows for memory cell independence one can apply memory and cache optimization without affecting the algorithm. The utility in this framework lies not only on its ability to speedup single sequence folding but its ability to speedup the family of RNA folding problems for which both Sparsification and Four-Russians have bene applied separately.

Future work in this area would be to examine the ability to sparsify memory [3], as Four-Russians at worst case requires an additional factor of $2^{\log (n)}$ in memory. Another open question is wether it is possible to apply the $\Omega\left(\log ^{3} n\right)$ [5] speedup of boolean matrix multiplication to RNA folding.

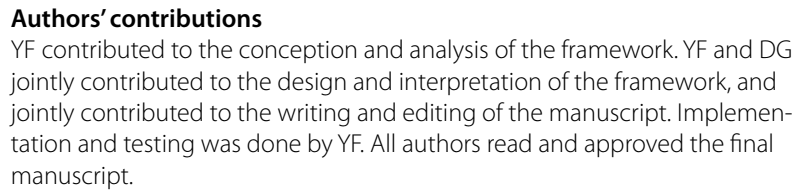
jointly contributed to the design and interpretation of the framework, and jointly contributed to the writing and editing of the manuscript. Implementation and testing was done by YF. All authors read and approved the final manuscript.

\section{Acknowledgements}

We would like to sincerely thank Shay Zakov and Michal Ziv-Ukelson for their their many helpful comments and suggestions. This research was partially supported by the IIS-1219278 Grant. uld like to sincerely thank Shay Zakov and Michal Ziv-Ukelson for their their many helpful comments and suggestions. This research was partially supported by the IIS-1219278 Grant.

\section{Competing interests}

The authors declare that they have no competing interests.

Received: 15 December 2015 Accepted: 28 June 2016

Published online: 05 August 2016

\section{References}

1. Akutsu T. Approximation and exact algorithms for RNA secondary structure prediction and recognition of stochastic context-free languages. J Comb Optim. 1999;3(2-3):321-36.

2. Andronescu M, Condon A, Hoos H, Mathews D, Murphy KP. Efficient parameter estimation for RNA secondary structure prediction. Bioinformatics. 2007;23(13):i19-28. doi: 10.1093/bioinformatics/btm223. http:// bioinformatics.oxfordjournals.org/content/23/13/i19.abstract

3. Backofen R, Tsur D, Zakov S, Ziv-Ukelson M (2009) Sparse RNA folding: time and space efficient algorithms. In: CPM09; 2009. p. 249-62

4. Backofen R, Tsur D, Zakov S, Ziv-Ukelson M. Sparse RNA folding: time and space efficient algorithms. J Discrete Algorithms. 2011;9(1):12-31. doi:10.1016/j.jda.2010.09.001.

5. Chan T. Speeding up the Four Russians algorithm by about one more logarithmic factor. In: SODA; 2015. p. 212-17

6. Do C, Woods D, Batzoglou S. Contrafold: RNA secondary structure prediction without physics-based models. Bioinformatics. 2006;22(14):e90-8. doi:10.1093/bioinformatics/btl246. http://bioinformatics.oxfordjournals. org/content/22/14/e90.abstract.

7. Dowell R, Eddy S. Evaluation of several lightweight stochastic contextfree grammars for RNA secondary structure prediction. BMC Bioinformatics. 2004;5(1):71. doi:10.1186/1471-2105-5-71. http://www.biomedcentral. com/1471-2105/5/71.

8. Durbin R, Eddy S, Krogh A, Mitchison G. Biological sequence analysis: probabilistic models of proteins and nucleic acids. Cambridge: Cambridge University Press; 1998. http://www.amazon.com/ Biological-Sequence-Analysis-Probabilistic-Proteins/dp/0521629713

9. Frid Y, Gusfield D. A simple, practical and complete $O\left(n^{3} / \log (n)\right)$-time algorithm for RNA folding using the four-Russians speedup. In: WABI; 2009. p. 97-107

10. Frid $Y$, Gusfield D. A simple, practical and complete $O\left(n^{3} / \log (n)\right)$-time algorithm for RNA folding using the [four-russians] speedup. Algorithms Mol Biol. 2010a;5(1):13.

11. Frid $Y$, Gusfield D. A worst-case and practical speedup for the RNA cofolding problem using the four-Russians idea. In: Moulton V, Singh M, editors. Algorithms in bioinformatics. Heidelberg: Springer; 2010b. p. 1-12.

12. Frid $Y$, Gusfield D. Speedup of RNA pseudoknotted secondary structure recurrence computation with the four-Russians Method. In: COCOA; 2012. p. $176-87$

13. Juan $V$, Wilson $C$. RNA Secondary structure prediction based on free energy and phylogenetic analysis. J Mol Biol. 1999;289(4):935-47.

14. Leontis NB, Westhof E. RNA 3D structure analysis and prediction. Berlin: Springer; 2012

15. Markham NR, Zuker M. UNAFold. In: Keith JM, editor. Bioinformatics, methods in molecular biology. New York: Humana Press; 2008. p. 3-31

16. Mathews D, Andre T, Kim J, Turner D, Zuker M. An updated recursive algorithm for RNA secondary structure prediction with improved thermodynamic parameters. Mol Modeling Nucleic Acids: 246-57

17. Mathews DH, Sabina J, Zuker M, Turner D. Expanded sequence dependence of thermodynamic parameters improves prediction of RNA secondary structure. J Mol Biol. 1999;288(5):911-40. doi:10.1006/ jmbi.1999.2700. http://www.sciencedirect.com/science/article/pii/ S0022283699927006.

18. Mathews DH, Disney MD, Childs J, Schroeder S, Zuker M, Turner DH Incorporating chemical modification constraints into a dynamic programming algorithm for prediction of RNA secondary structure. Proceedings of the National Academy of Sciences of the United States 
of America. 2004;101(19):7287-92. doi:10.1073/pnas.0401799101. http:// www.pnas.org/content/101/19/7287.abstract.

19. McCaskill JS. The equilibrium partition function and base pair binding probabilities for RNA secondary structure. Biopolymers. 1990;29(67):1 105-19. doi:10.1002/bip.360290621. http://dx.doi.org/10.1002/ bip.360290621.

20. Møhl M, Salari R, Will S, Backofen R, Sahinalp S. Sparsification of RNA structure prediction including pseudoknots. Algorithms Mol Biol. 2010;5:39.

21. Salari R, Will S, Backofen R, Sahinalp S, Möhl M. Sparsification of RNA structure prediction including pseudoknots. In: Moulton V, Singh M, editors. WABI. Berlin: Springer; 2010. p. 40-51.

22. Nussinov R, Jacobson A. Fast algorithm for predicting the secondary structure of single-stranded RNA. PNAS. 1980;77(11):6309-13. doi:10.1073/pnas.77.11.6309. http://dx.doi.org/10.1073/pnas.77.11.6309.

23. Nussinov R, Pieczenik G, Griggs JR, Kleitman DJ. Algorithms for loop matchings. SIAM J Applied Math. 1978;35(1):68-82. doi:10.1137/0135006. http://link.aip.org/link/?SMM/35/68/1.

24. Pinhas T, Zakov S, Tsur D, Ziv-Ukelson M. Efficient edit distance with duplications and contractions. Algorithms Mol Biol. 2013;8:27.

25. Reuter J, Mathews D. RNAstructure: software for RNA secondary structure prediction and analysis. BMC Bioinformatics. 2010;11(1):129. doi:10.1186/1471-2105-11-129. http://www.biomedcentral. com/1471-2105/11/129.

26. Salari R, Möhl M, Will S, Sahinalp S, Backofen R. Time and space efficient RNA-RNA interaction prediction via sparse folding. In: RECOMB; 2010. p. $473-90$

27. Sankoff D, Kruskal JB, Mainville S, Cedergreen R. Fast algorithms to determine RNA secondary structures containing multiple loops. In: Sankoff D, Kruskal JB, editors. Time warps, string edits and macromolecules: the theory and practice of sequence comparison. Boston: Addison-Wesley; 1983. p. 93-120.

28. Tinoco I, Borer P, Dengler B, Levine M, Uhlenbec O, Crothers D, Gralla J. Improved estimation of secondary structure in ribonucleic-acids. Nature. 1973;246(150):40-1.
29. Waterman MS, Smith TF. RNA secondary structure: a complete mathematical analysis. Math Biosc. 1978;42:257-66.

30. Wexler Y, Zilberstein CBZ, Ziv-Ukelson M. A study of accessible motifs and RNA folding complexity. J Comput Biol. 2007;14(6):856-72.

31. Williams R. Matrix-vector multiplication in sub-quadratic time: (some preprocessing required). In: Pruhs K, Stein C, editors. Bansal N. SIAM: SODA; 2007. p. 995-1001.

32. Williams R. Faster all-pairs shortest paths via circuit complexity. In: Symposium on theory of computing. STOC: New York; 2014. p. 664-73. doi:10.1145/2591796.2591811. http://doi.acm. org/10.1145/2591796.2591811

33. Xia T, SantaLucia J, Burkard M, Kierzek R, Schroeder S, Jiao X, Cox C, Turner D. Thermodynamic parameters for an expanded nearest-neighbor model for formation of RNA duplexes with watson-crick base pairs. Biochemistry. 1998;37(42):14,719-14,735. doi:10.1021/bi9809425. http://pubs.acs.org/ doi/abs/10.1021/bi9809425

34. Zakov S, Tsur D, Ziv-Ukelson M. Reducing the worst case running times of a family of RNA and CFG problems, using valiant's approach. In: WABl: 2010. p. 65-77

35. Ziv-Ukelson M, Gat-Viks I, Wexler Y, Shamir R. A faster algorithm for RNA Co-folding. In: Proceedings of the 8th International workshop on algorithms in bioinformatics. Waterville: WABI; 2008. p. 174-85

36. Zuker M. The use of dynamic programming algorithms in RNA secondary structure prediction. In: Waterman M, editor. Mathematical methods for DNA sequences. Boca Raton: CRC Press, Inc.; 1989. p. 159-84.

37. Zuker M. Mfold web server for nucleic acid folding and hybridization prediction. Nucleic Acids Res. 2003;31 (13):3406-3415. doi:10.1093/nar/ gkg595. http://nar.oxfordjournals.org/content/31/13/3406.full.pdf+html

38. Zuker M, Sankoff D. RNA secondary structures and their prediction. Bull Math Biol. 1984;46(4):591-621.

39. Zuker M, Stiegler P. Optimal computer folding of large RNA sequences using thermodynamics and auxiliary information. Nucleic Acids Res. 1981;9(1):133-48

\section{Submit your next manuscript to BioMed Central and we will help you at every step:}

- We accept pre-submission inquiries

- Our selector tool helps you to find the most relevant journal

- We provide round the clock customer support

- Convenient online submission

- Thorough peer review

- Inclusion in PubMed and all major indexing services

- Maximum visibility for your research

Submit your manuscript at www.biomedcentral.com/submit
() BioMed Central 